\title{
Ocular surface disease in patients with glaucoma or ocular hypertension treated with either BAK-preserved latanoprost or BAK-free travoprost
}

This article was published in the following Dove Press journal:

Clinical Ophthalmology

2 November 2010

Number of times this article has been viewed

\author{
Gregory Katz' \\ Clark L Springs ${ }^{2}$ \\ E Randy Craven ${ }^{3}$ \\ Michela Montecchi-Palmer ${ }^{4}$ \\ 'Huron Ophthalmology, Ypsilanti, MI, \\ USA; 'Indiana University Eye Care, \\ Indianapolis, IN, USA; ${ }^{3}$ Specialty \\ Eye Care, Denver, CO, USA; ${ }^{4}$ Alcon \\ Research Ltd., Fort Worth, TX, USA
}

Correspondence: Gregory Katz 5477 W. Clark Rd, Ypsilanti, MI 48197, USA

Tel +l 7344346000

Fax +I 7344347005

Email glbjkatz@hotmail.com
Purpose: The preservative benzalkonium chloride (BAK) may adversely affect ocular surface health. This study evaluated symptoms of ocular surface disease (OSD) in patients previously treated with a BAK-preserved therapy to lower their intraocular pressure, who either continued that therapy or switched to a BAK-free therapy.

Methods: Eligible adult patients with ocular hypertension or open-angle glaucoma that had been controlled with BAK-preserved latanoprost $0.005 \%$ monotherapy $\left(\right.$ Xalatan $\left.^{\circledR}\right)$ for at least one month and had a score of $\geq 13(0=$ none, $100=$ most severe $)$ on the Ocular Surface Disease Index (OSDI) questionnaire were entered into this prospective, double-masked, randomized, active-controlled, multicenter trial. By random assignment, patients either continued with BAK-preserved latanoprost $0.005 \%$ or transitioned to BAK-free travoprost $0.004 \%$ (Travatan $\mathrm{Z}^{\circledR}$ ophthalmic solution). OSDI scores were assessed again after six and 12 weeks.

Results: For the 678 evaluable patients, mean change in OSDI score from baseline to week 12 favored the travoprost $0.004 \%$ BAK-free group, but was not statistically different between groups $(P=0.10)$. When patients with mild OSD at baseline were assessed after 12 weeks, the mean OSDI score was significantly lower $(P=0.04)$ in the BAK-free travoprost $0.004 \%$ group ( score $=11.6 \pm 10.8$ units) than in the BAK-preserved latanoprost $0.005 \%$ group (score $=14.4 \pm 11.9$ units), and a significantly larger percentage $(P<0.01)$ improved to normal OSDI scores in the BAK-free travoprost $0.004 \%$ group $(62.9 \%$ of group) than in the BAKpreserved latanoprost $0.005 \%$ group ( $47.0 \%$ of group). Patients pretreated with BAK-preserved latanoprost $0.005 \%$ for $>24$ months were significantly more likely $(P=0.03)$ to improve to a normal OSDI score after 12 weeks if they were switched to BAK-free travoprost $0.004 \%(47.9 \%$ of group) than if they remained on BAK-preserved latanoprost $0.005 \%$ (33.9\% of group).

Conclusions: Switching from BAK-preserved latanoprost $0.005 \%$ to BAK-free travoprost $0.004 \%$ yielded significant improvements in symptoms of OSD in patients with glaucoma or ocular hypertension.

Keywords: ocular surface, glaucoma, benzalkonium chloride, prostaglandin analog, preservative

\section{Introduction}

Most of the currently available topical treatments for elevated intraocular pressure (IOP), including latanoprost $0.005 \%$, are preserved with benzalkonium chloride (BAK). ${ }^{1}$ Chronic exposure to BAK-preserved IOP-lowering medications has been associated with increased frequency of patient-reported symptoms of ocular discomfort, including burning, stinging, foreign body sensation, and dry eye sensation. ${ }^{2}$ In vitro, BAK-preserved latanoprost $0.005 \%$ and BAK-preserved travoprost $0.004 \%$ are both toxic to ocular cells, whereas BAK-free travoprost $0.004 \%$ is not. ${ }^{3}$ 
In animal models, BAK-free travoprost $0.004 \%$ did not affect goblet cell numbers ${ }^{4}$ or corneal epithelial cells, ${ }^{5,6}$ whereas BAK-preserved latanoprost $0.005 \%$ was shown to cause losses of goblet cells ${ }^{4}$ as well as pathologic changes in the corneal epithelium. ${ }^{5,6}$ In humans, chronic exposure to BAK-preserved topical IOP-lowering medications was associated with signs of adverse effects on the ocular surface, including instability of the tear film, ${ }^{7-9}$ reduced density of superficial epithelial cells, ${ }^{7}$ disruption of corneal epithelial barrier function, ${ }^{8}$ and conjunctival inflammation. ${ }^{9}$ Adverse reactions induced by BAK-preserved medications may be reversible in glaucoma patients who are switched to BAK-free medications. ${ }^{2}$ For these reasons, many researchers and clinicians have recommended BAKfree IOP-lowering medications. ${ }^{1-9}$

BAK-induced changes may manifest as symptomatic ocular surface disease (OSD) in medically treated glaucoma patients. ${ }^{10,11}$ OSD is an umbrella term that includes dry eye, lid disease, conjunctivitis, and keratitis. ${ }^{12}$ Although OSD is seen in approximately $15 \%$ of the general elderly population, ${ }^{13}$ it has been reported to occur in $48 \%$ to $59 \%$ of patients with medically treated glaucoma. ${ }^{10,11}$ A higher incidence $^{10}$ and severity ${ }^{11}$ of OSD has been reported in patients who received multiple BAK-preserved treatments concomitantly than in patients who were treated with only one BAK-preserved treatment. Antihypertensive medications with alternative preservative systems (other than BAK) could help to maintain the long-term ocular surface health of patients with glaucoma, and could avoid inducing or aggravating OSD.

In a previous large multicenter clinical trial of patients with glaucoma who had been previously treated with either latanoprost $0.005 \%$ or bimatoprost $0.03 \%$, and who needed alternative therapy due to tolerability issues, a switch to BAK-free travoprost $0.004 \%$ resulted in improvement in OSD symptoms that were both clinically and statistically significant, and maintained equal or better control of IOP. ${ }^{14}$ However, that study was not conducted in a parallel, randomized, masked fashion. The objective of this current multicenter, double-masked, randomized, controlled study was to quantify changes in symptoms of OSD after randomizing patients with open-angle glaucoma or ocular hypertension who were previously treated with latanoprost $0.005 \%$ preserved by $0.02 \%$ BAK (Xalatan ${ }^{\circledR}$ ophthalmic solution; Pfizer Inc., NY) either to remain on BAK-preserved latanoprost $0.005 \%$ or to change to BAK-free travoprost $0.004 \%$ (Travatan $\mathrm{Z}^{\circledR}$ ophthalmic solution; Alcon Laboratories, Inc., Fort Worth, TX).

\section{Methods}

This was a prospective, double-masked, randomized, activecontrolled clinical trial, conducted at 66 clinics in the US. The protocol was approved by the appropriate review boards at all participating institutions, and the trial was conducted in accordance with the tenets of the Declaration of Helsinki. All participating patients signed a written informed consent form.

\section{General entry criteria}

Eligible patients were at least 18 years of age, had ocular hypertension or primary open-angle glaucoma (with or without pigment dispersion or a pseudoexfoliation component), and had IOP that was adequately controlled on latanoprost $0.005 \%$ monotherapy for at least one month prior to enrollment. Adequate IOP control was determined for each patient by the enrolling investigator, and was defined as being both stable and safe for that patient. General ocular health exclusion criteria included the following: corneal abnormalities that could prevent accurate applanation tonometry; any intraocular surgery or ocular trauma within the previous six months; any ocular laser surgery within the previous three months; progressive retinal or optic nerve disease; severe central visual field loss; or visual acuity worse than $0.6 \log$ MAR in either eye. Patients were also excluded if they had used any ocular medications (other than latanoprost $0.005 \%$ or artificial tears) within seven days of the screening visit, or if they had taken any systemic medication for less than 30 days of stable dosing before the screening visit. Women of childbearing potential were allowed to participate in the trial only if they were not breastfeeding, were not pregnant or planning to become pregnant, and were using adequate birth control during the study. All patients were required to be willing and able to abstain from the use of any other topical ophthalmic eye drops, other than assigned study medication, for the duration of the study.

\section{Ocular surface entry criteria}

Exclusion criteria related to ocular surface health were as follows: OSD that had previously been treated with punctal plugs, punctal cautery, topical cyclosporine A, or topical corticosteroids; suspected or diagnosed Sjögren's syndrome; prior corneal surgery (including keratorefractive surgery) within the previous one year; presence or history of clinically significant blepharitis within the previous two years; any history of other ocular inflammatory disease (eg, rosacea that affected the ocular adnexa or herpes 
simplex virus keratitis); seasonal ocular allergies expected within the study period; and any contact lens wear or corticosteroid use within the 30 days before the screening visit.

All potential patients were screened using the Ocular Surface Disease Index (OSDI) questionnaire. The OSDI is a validated, self-administered instrument for assessing the presence and severity of OSD symptoms. ${ }^{15}$ The OSDI questionnaire includes 12 questions about the respondent's past-week experience with ocular symptoms, vision-related functioning, and environmental triggers. ${ }^{15,16}$ Questions assessed whether respondents had eyes that felt gritty, painful, sore, or sensitive to light; whether they had blurred or poor vision; whether they experienced limitations with reading, driving at night, watching television, or working with a computer or bank machine; and whether their eyes felt uncomfortable in windy conditions, in areas with low humidity, or in air-conditioned places. ${ }^{16}$ Response options for each question were "all of the time" ( score $=4)$, "most of the time" ( score $=3$ ), "half of the time" ( score $=2)$, "some of the time" ( score $=1)$, and "none of the time" (score $=0) .{ }^{15}$ Questions about vision-related functioning or environmental triggers could also be answered with "not applicable", in which case that question was not factored into the final score calculation. The total OSDI score was calculated for each patient using the methods described by the OSDI originators, ${ }^{15}$ as follows:

$$
\text { OSDI score }=\frac{(\text { sum } \text { of scores for all questions answered }) \times 25}{\text { Total number of questions answered }}
$$

The final total OSDI score could range from 0 to 100 , with the OSDI scores classified as $\leq 12=$ normal, $13-22=$ mild OSD, 23-32 = moderate OSD, and $\geq 33=$ severe OSD. ${ }^{17}$ To be eligible for inclusion in the study, patients were required to have an OSDI score of 13 or higher.

All potential patients were screened with corneal fluorescein staining. Corneal fluorescein staining was conducted according to each investigator's standard procedure, using their standard staining agent. Each cornea was scored on the following scale, which was designed to assess staining over the entire corneal surface with no specification of corneal regions: $0=$ absent (no staining), $1=$ mild (a few punctate regions of staining, but less than $10 \%$ coverage of the corneal surface), $2=$ moderate $(10 \%-50 \%$ coverage of the corneal surface), or $3=$ severe (more than $50 \%$ coverage of the corneal surface). To be eligible for the study, patients were required to have a corneal fluorescein staining score of 1 or higher.

\section{Enrollment and masked randomization}

Patients who met all entry criteria and who reported using latanoprost $0.005 \%$ on the evening prior to the screening/enrollment visit were invited to participate. At the enrollment visit, the informed consent form was signed, comprehensive medical and ophthalmic histories were obtained, the OSDI questionnaire was completed, and an ocular examination was performed (including visual acuity determination, slit-lamp inspection of the anterior segment, corneal fluorescein staining, Goldmann tonometry, and dilated fundus examination). Women of childbearing potential provided urine samples for pregnancy tests.

The targeted enrollment was approximately 700 patients (350 per group) in order to obtain approximately 650 evaluable patients (325 per group). Power calculations (using a two-sample $t$-test with two-sided alpha $=0.05$ ) indicated that, with 325 patients per treatment group, the study would have at least $90 \%$ power to detect a difference between treatment groups that was $>5$ units on the OSDI questionnaire, assuming a common standard deviation of 19 units in the mean change from baseline OSDI score.

At the completion of the first visit, enrolled patients were randomized to an intervention whereby they either continued on therapy with BAK-preserved latanoprost $0.005 \%$ or were transitioned to therapy with BAK-free travoprost $0.004 \%$ ophthalmic solution. BAK-preserved latanoprost $0.005 \%$ was the commercially available Xalatan ${ }^{\circledR}$ ophthalmic solution, which is preserved using $0.02 \% \mathrm{BAK}$. BAK-free travoprost $0.004 \%$ was the commercially available Travatan $Z^{\circledR}$ ophthalmic solution containing the proprietary sofZia ${ }^{\circledR}$ preservative, which is an ionic buffer system containing borate, propylene glycol, sorbitol, and zinc chloride.

If both eyes met all of the eligibility criteria, both eyes were treated with the same test medication; otherwise, only the eligible eye was treated. At the study site, the enrolling clinician assigned a number to the patient, and then called an interactive voice response system that was hosted by the study sponsor in order to receive a kit number. These kit numbers had been randomized by the study sponsor using statistical software (SAS Institute, Cary, NC). The patient received the assigned kit of study medication. Within the kits, all medications (whether BAK-preserved latanoprost $0.005 \%$ or BAK-free travoprost $0.004 \%$ ) were packaged in identical oval $4 \mathrm{~mL}$ polypropylene dropper bottles. Each patient received two bottles of the assigned study medication and was instructed to instill one drop of study medication in the study eye(s) once daily in the evening. In case of a medical emergency that required information about the study 
medication, the investigator or a designee could request unmasking of the test medication by calling the interactive voice response system.

\section{Efficacy and safety assessments}

Patients returned six weeks (42 \pm 7 days) after enrollment for the second study visit, and 12 weeks ( $90 \pm 7$ days) after enrollment for the third study visit. Both of these visits were scheduled at approximately the same time of day as the entry visit for each patient. At both follow-up visits, an interval medical history was obtained and any adverse events were assessed, the OSDI questionnaire was completed, and an ocular examination was conducted, consisting of visual acuity, slit-lamp anterior segment inspection, corneal fluorescein staining, and Goldmann tonometry. At the 12-week visit, a dilated fundus examination was conducted, and women of childbearing potential provided urine samples for pregnancy tests.

The primary efficacy variable was the mean change in OSDI scores between the entry visit and the 12-week follow-up visit. A secondary efficacy variable was the percentage of patients with a corneal fluorescein staining score of 0 . Exploratory efficacy variables that were assessed at

Table I Baseline values and demographics for the intent-to-treat population

\begin{tabular}{|c|c|c|c|}
\hline & $\begin{array}{l}\text { Travoprost } \\
0.004 \% \text { BAK-free, } \\
n=343\end{array}$ & $\begin{array}{l}\text { BAK-preserved } \\
\text { latanopros } \\
0.005 \%, n=335\end{array}$ & $\begin{array}{l}\text { Overall } \\
\text { population } \\
n=678\end{array}$ \\
\hline \multicolumn{4}{|l|}{ Age, n (\%) } \\
\hline $18-64$ years & $|3|(38.2 \%)$ & II 4 (34.0\%) & $245(36.1 \%)$ \\
\hline$\geq 65$ years & $212(61.8 \%)$ & $221(66.0 \%)$ & $433(63.9 \%)$ \\
\hline \multicolumn{4}{|c|}{ Gender, n (\%) } \\
\hline Male & I 32 (38.5\%) & $105(31.3 \%)$ & 237 (35.0\%) \\
\hline Female & $211(61.5 \%)$ & $230(68.5 \%)$ & $44 \mathrm{I}(65.0 \%)$ \\
\hline \multicolumn{4}{|c|}{ OSDI category, n (\%) } \\
\hline Normal* & $2(0.6 \%)$ & $2(0.6 \%)$ & $4(0.6 \%)$ \\
\hline Mild & $|4|(4 \mid .1 \%)$ & $136(40.6 \%)$ & 277 (40.9\%) \\
\hline Moderate & $91(26.5 \%)$ & $85(25.4 \%)$ & $176(26.0 \%)$ \\
\hline Severe & $109(31.8 \%)$ & $112(33.4 \%)$ & $221(32.6 \%)$ \\
\hline \multicolumn{4}{|c|}{ Duration of BAK-preserved latanoprost pretreatment, n (\%) } \\
\hline $\begin{array}{l}\text { Total with } \\
\text { data available }\end{array}$ & $311(90.7 \%)$ & $300(89.6 \%)$ & $611(90.1 \%)$ \\
\hline $\begin{array}{l}\text { Without } \\
\text { data available }\end{array}$ & $32(9.3 \%)$ & $35(10.4 \%)$ & 67 (9.9\%) \\
\hline I-6 months & $109(31.8 \%)$ & $100(29.9 \%)$ & $209(30.8 \%)$ \\
\hline 6-24 months & $78(22.7 \%)$ & $87(26.0 \%)$ & $165(24.3 \%)$ \\
\hline$>24$ months & $124(36.2 \%)$ & $113(33.7 \%)$ & $237(35.0 \%)$ \\
\hline
\end{tabular}

Notes: *These patients were enrolled due to an error in calculating the baseline OSDI score; † Of those patients who could recall their start date with BAK-preserved latanoprost $0.005 \%$.

Abbreviations: OSDI, Ocular Surface Disease Index; BAK, benzalkonium chloride. the 12-week follow-up visit were the proportion of patients who had a normal OSDI score ( $\leq 12$ units), percentage of patients with $\mathrm{a} \geq 10$-point improvement from baseline OSDI score, OSDI outcomes stratified by duration of pretreatment with BAK-preserved latanoprost $0.005 \%$ before entering the study, and OSDI outcomes stratified by severity of baseline OSDI (mild, moderate, or severe). Safety variable assessments included best-corrected visual acuity, slit lamp evaluations, IOP, dilated fundus examinations, and adverse events.

\section{Statistical analysis}

Continuous variables were assessed using a two-sample $t$-test with two-sided $\alpha=0.05$, and categoric variables were assessed by Chi-square test with $\alpha=0.05$. The null hypothesis stated that no relationship existed between BAK-preserved treatment and change in OSDI score. The alternative hypotheses stated that BAK-preserved latanoprost $0.005 \%$ had an adverse impact on OSD, which might accrue over a longer duration of BAKpreserved latanoprost $0.005 \%$ treatment, but might be reversible to some degree by transition to BAK-free travoprost $0.004 \%$, especially in patients with mild OSDI. Unless otherwise specified, outcome values are presented as mean \pm standard deviation in text, and as mean \pm standard error in figures.

\section{Results}

\section{Baseline clinical and demographic data}

A total of 724 patients were enrolled, 678 of whom were evaluable for the intent-to-treat (ITT) analysis. Four of the ITT patients had normal OSDI scores at baseline, which was an exclusion criterion, but they received study medication and thus were evaluated with the rest of the population. As shown in Table 1, the two treatment groups were statistically similar (all $P>0.05$ ) in the baseline parameters of gender, age, OSDI category, and duration of exposure to BAK-preserved latanoprost $0.005 \%$ before entry into the study. The first visit of the first patient was in July 2008, and the final analysis date was in June 2009. Participant flow through the study is shown in Figure 1.

\section{Mean change in OSDI scores}

For the patients who had mild OSD at baseline, the mean OSDI score at the 12-week time point was significantly lower $(P=0.04)$ in patients randomized to BAK-free travoprost $0.004 \%$ (11.6 \pm 10.8 units) than in patients who continued on BAK-preserved latanoprost $0.005 \%$ (14.4 \pm 11.9 units), as shown in Figure 2. For the overall cohort of patients with all baseline OSDI scores, mean OSDI scores at the 12-week 


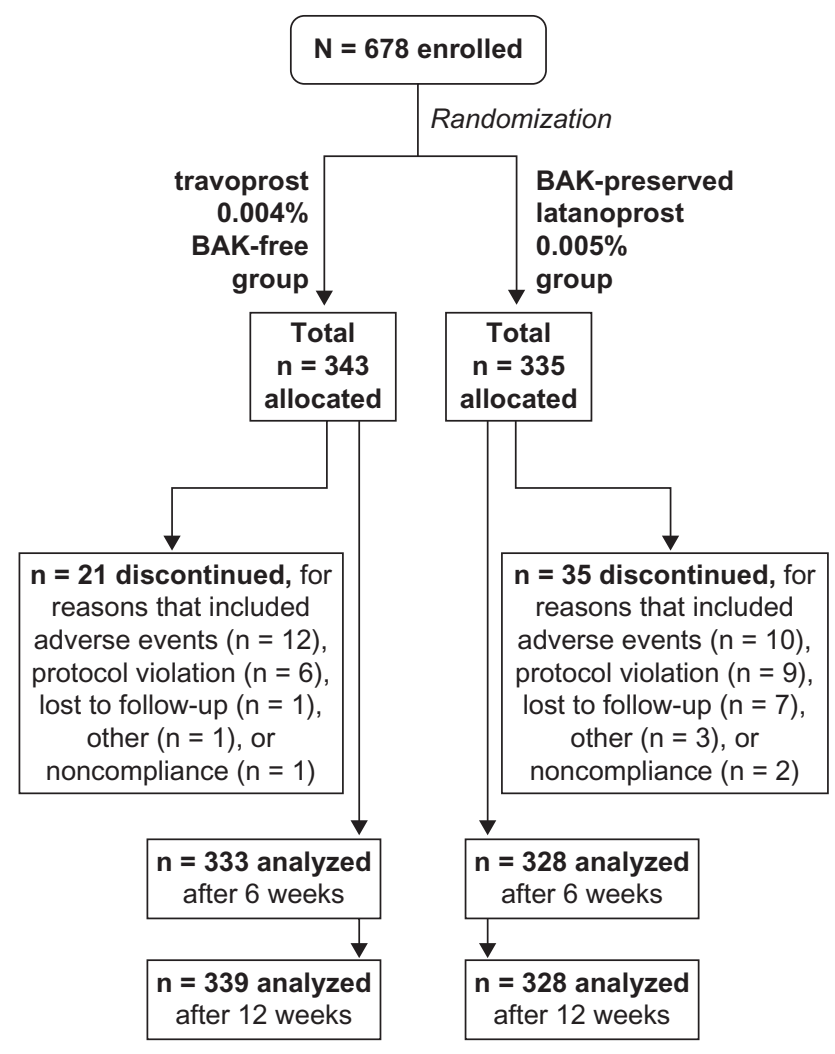

Figure I Participant flow through the study. When possible, patients who discontinued treatment were analyzed before exiting the study, so discontinuation and analysis numbers are not mutually exclusive.

Abbreviation: BAK, benzalkonium chloride.

time point were not statistically different between the groups, ie, $18.4 \pm 16.0$ for 339 patients in the BAK-free travoprost $0.004 \%$ group, and $19.4 \pm 15.3$ for 328 patients in the BAKpreserved latanoprost $0.005 \%$ group.

When normalized to baseline values, the mean change in OSDI score from the entry visit to the 12-week follow-up visit was not significantly larger $(P=0.10)$ for the patients with mild OSD at baseline who were randomized to BAK-free travoprost $0.004 \%(-5.0 \pm 10.8$ units, $n=140)$ than for patients with mild OSD at baseline who continued on BAK-preserved latanoprost $0.005 \%(-2.7 \pm 12.1$ units, $n=132)$, as shown in Figure 3. The mean change from baseline mild OSDI score to score at week 12 was statistically different, from zero change in both treatment groups $(P=0.01$ in the BAK-preserved latanoprost $0.005 \%$ group and $P<0.0001$ in the BAK-free travoprost $0.004 \%$ group). Mean change from baseline OSDI scores in the "baseline-moderate" and "baseline-severe" groups were not statistically different between the treatment groups. For the overall cohort of patients with all baseline OSDI scores, mean changes in OSDI scores at the 12-week time point were not statistically different between groups $(-11.3 \pm 17.2$ for the 339 patients in the BAK-free travoprost

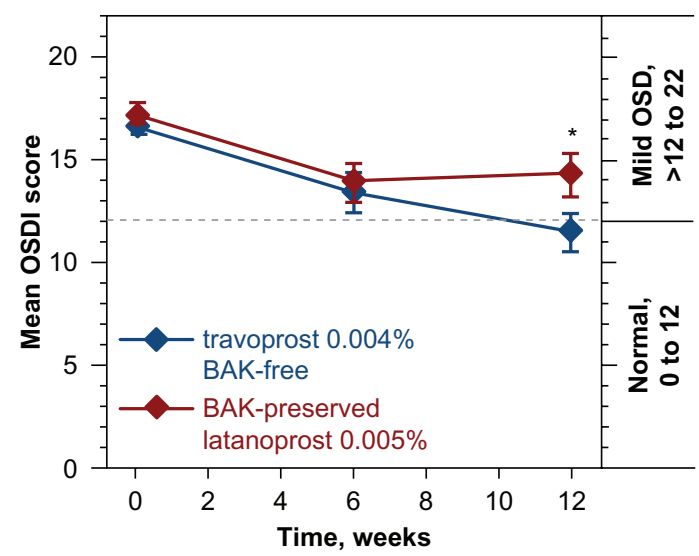

Figure 2 Mean scores on the OSDI questionnaire for the patients who had mild OSD at baseline. Error bars represent standard error of the mean. $* P<0.05$. In the BAK-free travoprost group, patient numbers were $|4|$ at baseline, 135 at week 6 , and 140 at week 12. In the BAK-preserved latanoprost group, patient numbers were 136 at baseline, 134 at week 6 , and I 32 at week 12 .

Abbreviations: BAK, benzalkonium chloride: OSDI, Ocular Surface Disease Index; OSD, ocular surface disease.

$0.004 \%$ group and $-11.4 \pm 17.4$ for the 328 patients in the BAK-preserved latanoprost $0.005 \%$ group).

\section{Patients improving to normal OSDI scores}

The percentage of patients who had mild OSDI scores at baseline and who improved to normal OSDI scores after 12 weeks was significantly larger $(P<0.01)$ in the BAK-free travoprost

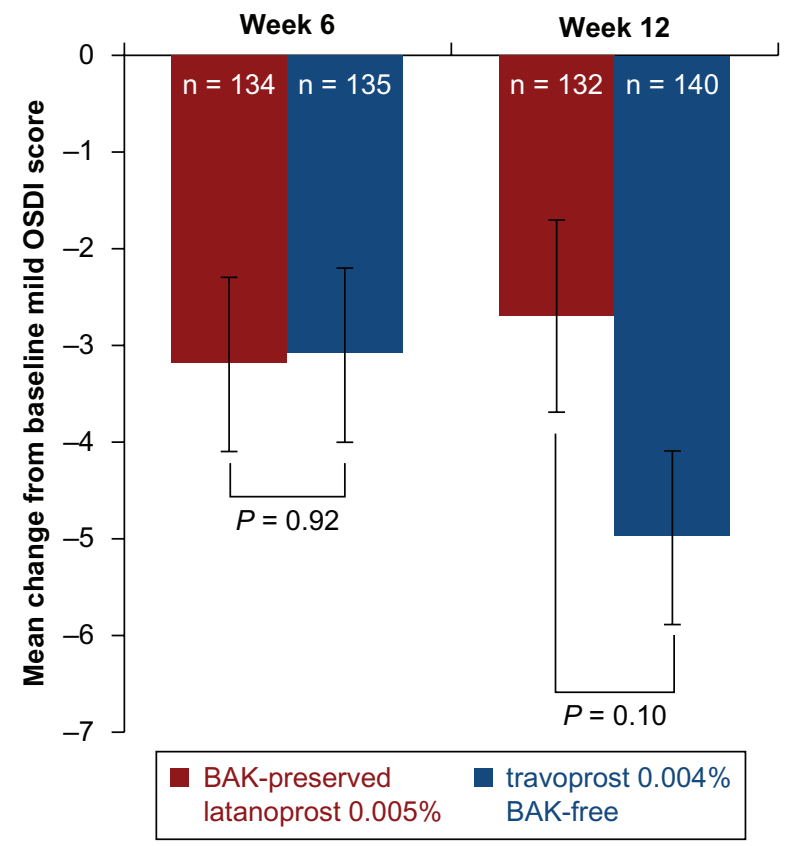

Figure 3 Mean change from baseline scores on the OSDI questionnaire for the patients who had mild ocular surface disease at baseline. Error bars represent standard error of the mean.

Abbreviations: BAK, benzalkonium chloride; OSDI, Ocular Surface Disease Index. 
$0.004 \%$ group $(62.9 \%$ of group, 88 of 140$)$ than in the BAKpreserved latanoprost $0.005 \%$ group (47.0\% of group, 62 of 132), as shown in Figure 4. Percentages of those patients who had started at "baseline-moderate" or "baseline-severe" OSDI scores and who improved to normal OSDI scores at 12 weeks were not significantly different between the treatment groups. For the overall cohort of patients with all baseline OSDI scores, the percentage of patients who improved to normal OSDI scores after 12 weeks was not significantly different between groups, ie, $41.6 \%$ of patients (141 of 339) in the BAK-free travoprost $0.004 \%$ group, and $38.4 \%$ of patients (126 of 328) in the BAK-preserved latanoprost $0.005 \%$ group.

\section{Outcomes stratified by duration of pretreatment}

Of the evaluable population, 611 patients could recall how long they had been pretreated with BAK-preserved latanoprost before entering the study. Prior exposure to BAK-preserved latanoprost was stratified as $>24$ months in $35.0 \%$ of patients (237 of 678), 6-24 months in $24.3 \%$ of patients (165 of 678), $1-6$ months in $30.8 \%$ of patients (209 of 678 ), and of unknown duration in $9.9 \%$ of patients (67 of 678), as shown in Table 1. Regardless of baseline OSDI score, patients who were pretreated with BAK-preserved latanoprost $0.005 \%$ for $>24$ months before entering the

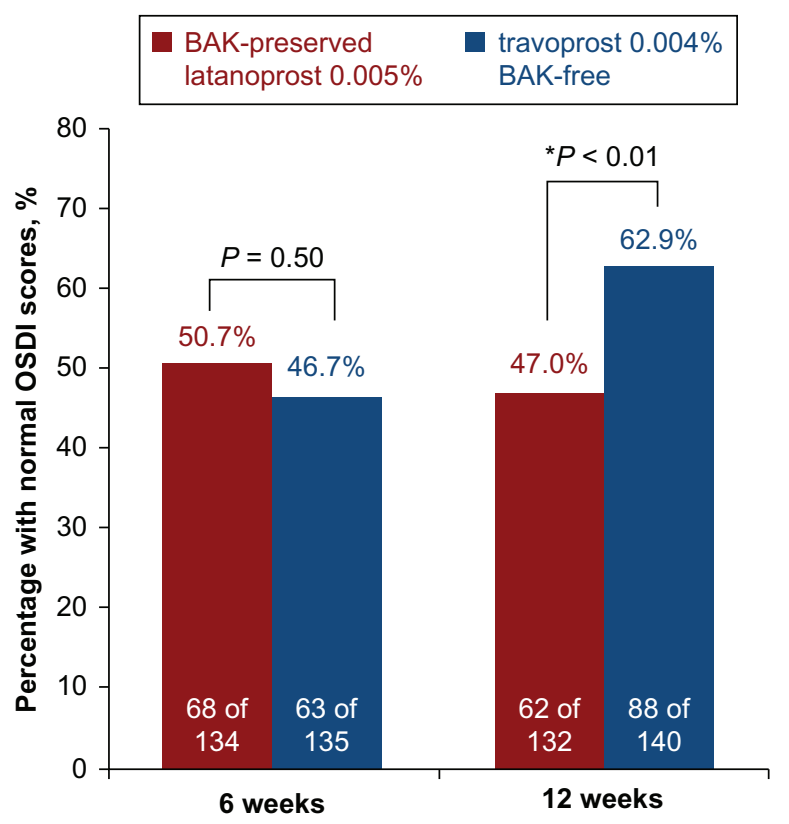

Figure 4 Percentage of patients who had baseline mild-severity scores on the OSDI and who improved to normal OSDI scores.

Abbreviations: BAK, benzalkonium chloride OSDI, Ocular Surface Disease Index. study were significantly more likely $(P=0.03)$ to improve to a normal OSDI score after 12 weeks if they were switched to BAK-free travoprost $0.004 \%$ ( $47.9 \%$ of patients, 58 of 121 ) than if they remained on BAK-preserved latanoprost $0.005 \%$ (33.9\% of patients, 37 of 109), as shown in Figure 5. The percentage of patients improving to a normal OSDI score was not significantly different between the treatment groups for patients who were exposed to BAK-preserved latanoprost $0.005 \%$ for $1-6$ months or for $6-24$ months prior to entry into the study.

\section{Patients with $\geq 10$ point improvement in OSDI scores}

The percentage of patients who improved $\geq 10$ points in OSDI scores from baseline to week 12 was not statistically different between the treatment groups for the overall cohort, or for the subgroups of patients with mild, moderate, or severe baseline OSDI scores. For the overall population, an improvement of $\geq 10$ points was observed in $53.4 \%$ of patients (181 of 339) in the BAK-free travoprost $0.004 \%$ group and in $51.8 \%$ of patients (170 of 328) in the BAKpreserved latanoprost $0.005 \%$ group.

\section{Absence of corneal staining}

The percentage of patients without corneal staining at week 12 was not statistically different between treatment groups

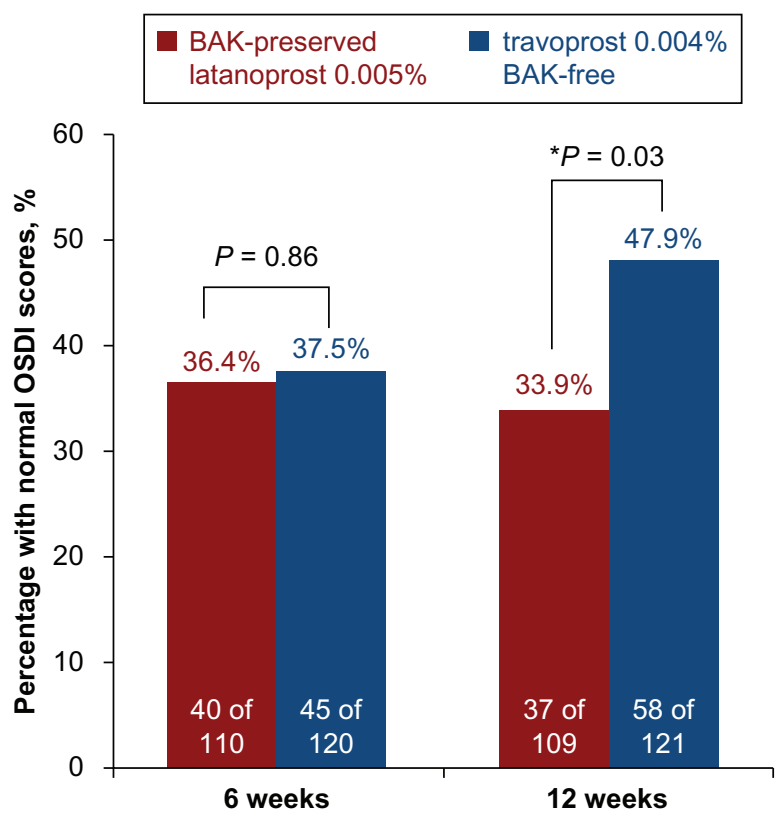

Figure 5 Percentage of patients who had been exposed to BAK-preserved latanoprost for $>24$ months prior to entry in the study, and who improved to normal scores on the OSDI 12 weeks after switching to BAK-free travoprost. Abbreviations: BAK, benzalkonium chloride; OSDI, Ocular Surface Disease Index. 
for the overall cohort, or for the subgroups of patients with mild, moderate, or severe baseline OSDI scores. For the overall population, absence of corneal staining at week 12 was observed in $37.1 \%$ of patients (127 of 342 ) in the BAK-free travoprost $0.004 \%$ group and $40.0 \%$ of patients (132 of 330 ) in the BAK-preserved latanoprost $0.005 \%$ group.

\section{Safety assessments}

No statistical differences in safety parameters were observed between treatment groups. The entire enrolled population, not just the ITT population, was assessed for safety parameters. The most frequently reported treatment-emergent ocular adverse events in both treatment groups were eye irritation, hyperemia of the eye, eye pain, eye pruritus, and foreign body sensation in the eye, as shown in Table 2.

\section{Discussion}

In this randomized, controlled, multicenter, 12-week study of 678 glaucoma patients who had been treated with BAKpreserved latanoprost $0.005 \%$ for at least one month prior to entry to the study, transitioning to BAK-free travoprost $0.004 \%$ produced significant improvements in symptoms of OSD for patients who had mild OSDI scores at baseline and for patients who had been exposed to BAK-preserved latanoprost $0.005 \%$ for more than 24 months prior to entry into the study.

For patients with mild OSDI scores at baseline, the mean improvement was -5.0 units on the OSDI questionnaire for patients who were transitioned to BAK-free travoprost $0.004 \%$. This value (5.0 units) is clinically relevant, because it has been established that the minimum clinically important difference in OSDI score is 4.5 units in patients with mild or moderate OSD. ${ }^{17}$ The patients in this study who continued on BAK-preserved latanoprost $0.005 \%$ had OSDI scores that improved by only 2.7 points, which did not meet

Table 2 Most frequently reported treatment-emergent ocular adverse events

\begin{tabular}{|c|c|c|}
\hline & $\begin{array}{l}\text { BAK-free } \\
\text { travoprost } 0.004 \% \\
n=362\end{array}$ & $\begin{array}{l}\text { BAK-preserved } \\
\text { latanoprost } 0.005 \% \\
\mathbf{n = 3 6 2}\end{array}$ \\
\hline Eye irritation, n (\%) & $9(2.5 \%)$ & $4(1.1 \%)$ \\
\hline $\begin{array}{l}\text { Hyperemia of } \\
\text { the eye, } n(\%)\end{array}$ & II (3.0\%) & $4(1.1 \%)$ \\
\hline Eye pain, n (\%) & $5(1.4 \%)$ & $2(0.6 \%)$ \\
\hline Eye pruritus, n (\%) & $3(0.8 \%)$ & $4(1.1 \%)$ \\
\hline $\begin{array}{l}\text { Foreign body } \\
\text { sensation in } \\
\text { eyes, n (\%) }\end{array}$ & $2(0.6 \%)$ & $4(1.1 \%)$ \\
\hline
\end{tabular}

that established minimum clinically important difference requirement.

Of patients with mild OSDI scores at baseline, those who were transitioned to BAK-free travoprost $0.004 \%$ were significantly more likely to return to normal OSDI scores at week 12 and had significantly lower mean scores on the OSDI at week 12, when compared with the patients who remained on BAK-preserved latanoprost $0.005 \%$. These outcomes confirm the validity of the recommendation to switch to BAK-free therapy that has been advocated by researchers who have investigated BAK-preserved IOP-lowering medications in vitro, ${ }^{3,18}$ in animals, ${ }^{4-6}$ and in glaucoma patients. ${ }^{7-9,19}$ Because reversal of the corneal epithelial cell layer damage in glaucoma patients who were treated chronically with BAKpreserved medications would occur gradually over time, patients with moderate or severe OSD may need more than the 12 weeks allotted in this study before significant OSD improvements could be observed after switching to BAKfree travoprost $0.004 \%$. Patients with moderate or severe OSD may have had more factors influencing their ocular surface health than the BAK insult alone; ie, these patients may have had underlying dry eye pathology or other factors that compounded the effect of BAK. In such cases, removing BAK would be helpful, but not sufficient, in ameliorating the condition.

Another important finding of this study was that patients who had been exposed to BAK-preserved latanoprost $0.005 \%$ for more than 24 months prior to entry into the study were significantly more likely to improve to normal OSDI scores if they were transitioned to BAK-free travoprost $0.004 \%$ than if they remained on BAK-preserved latanoprost $0.005 \%$. The same was not true of patients pretreated with BAK-preserved latanoprost $0.005 \%$ for 24 months or less. These results may indicate some cumulative, long-term adverse effect of BAK on ocular surface health, as has been suggested by in vitro studies demonstrating that BAK has dose-dependent toxic effects, ${ }^{20-22}$ and by animal studies demonstrating that BAK is persistent in ocular tissues over time. ${ }^{23}$ Together, these preclinical studies indicate that long-term buildup of daily doses of BAK in glaucoma patients could have dose-dependent toxic effects on ocular surface health. Such long-term exposure may be common among patients with glaucoma or ocular hypertension: one large-scale epidemiologic study found that patients had been medically treated for a median of 3.9 years. ${ }^{2}$ For the patients who were exposed to BAK-preserved latanoprost $0.005 \%$ for more than 24 months before entering this study, the effects of long-term BAK exposure on OSD were reversible within 12 weeks for some patients after switching 
to BAK-free travoprost $0.004 \%$. Similar reversibility of adverse ocular signs and symptoms has been demonstrated with therapeutic switches to BAK-free IOP-lowering treatments elsewhere in the literature. ${ }^{2,19}$

Some of the improvements that were observed in this study may be attributable to regression towards the mean, to increased familiarity with the OSDI questionnaire, or to placebo effects. ${ }^{24}$ However, such effects seemed to occur equally in both treatment groups up to the first follow-up visit at week 6 , but then separation between groups occurred between week 6 and week 12 (see Figure 2). Placebo effects may also partly explain the lack of difference between the treatment groups in corneal staining (in that case, the placebo effect would involve the investigators seeing what they expected to see). Alternatively, or in addition, the staining scale that was used in this study may not have been sensitive enough to distinguish changes in staining between the groups, and the categories on the staining scale may have been too broad. Patient-reported outcomes should be considered to be more important than corneal staining in this study, because the OSDI questionnaire reflects the patient's perception of their quality of life related to ocular symptoms, and because discordance often exists between clinical signs and ocular symptoms of OSD in individual patients. ${ }^{25}$ Overall, the improvements in OSDI scores that were observed at week 12 for patients who were transitioned to BAK-free travoprost $0.004 \%$ can be attributed directly to the change in therapy, after most methodologic effects were cancelled out earlier in the study (at week 6).

In addition to the way that a placebo effect may have increased subjective relief, a washout from a possible anesthetic effect of BAK may have increased subjective discomfort. An esthesiometry study has demonstrated that control groups and preservative-free groups had significantly more sensitivity in their corneas than did groups of patients who were treated with BAK-preserved IOP-lowering therapies; ${ }^{7}$ ie, BAK may act as an anesthetic, and may mask the experience of subjective symptoms associated with an underlying pathology. In switching to a BAK-free therapy, the loss of this hypoesthetic effect of BAK may allow some previously masked discomfort to be experienced. As the health of the ocular surface improves after a switch to the BAK-free treatment, an anesthetic effect would become less useful with time, and especially when the long-term benefits of a BAK-free treatment have been fully realized.

The findings of this study have important implications for the medical management of patients with glaucoma, because OSD is common among glaucoma patients, ${ }^{10}$ and because glaucoma patients have identified tolerability issues as barriers to compliance with IOP-lowering therapies. ${ }^{26}$ In choosing an IOP-lowering medication for glaucoma or ocular hypertension, a physician should consider both efficacy and tolerability. The IOP-lowering efficacy is equivalent between BAK-free travoprost $0.004 \%$ and BAK-preserved travoprost $0.004 \% .{ }^{27}$ Therefore, physicians could reasonably consider a switch from a BAK-preserved therapy to BAKfree travoprost $0.004 \%$ in patients with ongoing glaucoma who have comorbid OSD, or could consider initiating BAK-free travoprost $0.004 \%$ in new glaucoma patients who are at risk of developing OSD due to use of BAK-preserved products.

In summary, this was a randomized, controlled, multicenter, 12-week study of 678 glaucoma patients who had been treated with BAK-preserved latanoprost $0.005 \%$ for at least one month prior to entry to the study, and the results indicated that transitioning to BAK-free travoprost $0.004 \%$ yielded significant improvements in symptoms of OSD for patients who had mild OSDI scores at baseline and for patients who had been exposed to BAK-preserved latanoprost for more than 24 months prior to entry into the study. These observations support the conclusion that BAK-free travoprost $0.004 \%$ is superior to BAK-preserved latanoprost $0.005 \%$ in restoring a healthy ocular surface in glaucoma patients with OSD who require treatment for reduction of IOP.

\section{Disclosure}

This study was funded by Alcon Research Ltd, which also provided the assistance of a medical writer.

\section{References}

1. Yee RW. The effect of drop vehicle on the efficacy and side effects of topical glaucoma therapy: A review. Curr Opin Ophthalmol. 2007; 18(2):134-139.

2. Pisella PJ, Pouliquen P, Baudouin C. Prevalence of ocular symptoms and signs with preserved and preservative free glaucoma medication. $\mathrm{Br} J$ Ophthalmol. 2002;86(4):418-423.

3. Baudouin C, Riancho L, Warnet JM, Brignole F. In vitro studies of antiglaucomatous prostaglandin analogues: Travoprost with and without benzalkonium chloride and preserved latanoprost. Invest Ophthalmol Vis Sci. 2007;48(9):4123-4128.

4. Kahook MY, Noecker R. Quantitative analysis of conjunctival goblet cells after chronic application of topical drops. Adv Ther. 2008; 25(8):743-751.

5. McCarey B, Edelhauser H. In vivo corneal epithelial permeability following treatment with prostaglandin analogs [correction of analoges] with or without benzalkonium chloride. J Ocul Pharmacol Ther. 2007; 23(5):445-451.

6. Whitson J, Cavanagh H, Lakshman N, Petroll W. Assessment of corneal epithelial integrity after acute exposure to ocular hypotensive agents preserved with and without benzalkonium chloride. Adv Ther. 2006;23(5):663-671. 
7. Martone G, Frezzotti P, Tosi GM, et al. An in vivo confocal microscopy analysis of effects of topical antiglaucoma therapy with preservative on corneal innervation and morphology. Am J Ophthalmol. 2009;147(4):725-735.

8. Ishibashi T, Yokoi N, Kinoshita S. Comparison of the short-term effects on the human corneal surface of topical timolol maleate with and without benzalkonium chloride. J Glaucoma. 2003;12(6): 486-490.

9. Horsley MB, Kahook MY. Effects of prostaglandin analog therapy on the ocular surface of glaucoma patients. Clin Ophthalmol. 2009; 3:291-295

10. Leung EW, Medeiros FA, Weinreb RN. Prevalence of ocular surface disease in glaucoma patients. J Glaucoma. 2008;17(5):350-355.

11. Fechtner RD, Godfrey DG, Budenz D, Stewart JA, Stewart WC, Jasek MC. Prevalence of ocular surface complaints in patients with glaucoma using topical intraocular pressure-lowering medications. Cornea. 2010;29(6):618-621.

12. The International Dry Eye WorkShop Study Group. Methodologies to diagnose and monitor dry eye disease: Report of the Diagnostic Methodology Subcommittee of the International Dry Eye WorkShop (2007). Ocul Surf. 2007;5(2):108-152.

13. Schein OD, Munoz B, Tielsch JM, Bandeen-Roche K, West S. Prevalence of dry eye among the elderly. Am J Ophthalmol. 1997;124(6): 723-728

14. Henry JC, Peace JH, Stewart JA, Stewart WC. Efficacy, safety, and improved tolerability of travoprost BAK-free ophthalmic solution compared with prior prostaglandin therapy. Clin Ophthalmol. 2008;2(3):613-621.

15. Schiffman RM, Christianson MD, Jacobsen G, Hirsch JD, Reis BL. Reliability and validity of the Ocular Surface Disease Index. Arch Ophthalmol. 2000;118(5):615-621.

16. Ozcura F, Aydin S, Helvaci MR. Ocular surface disease index for the diagnosis of dry eye syndrome. Ocul Immunol Inflamm. 2007;15(5):389-393.

17. Miller KL, Walt JG, Mink DR, et al. Minimal clinically important difference for the ocular surface disease index. Arch Ophthalmol. 2010;128(1):94-101.
18. Kahook MY, Ammar DA. In vitro toxicity of topical ocular prostaglandin analogs and preservatives on corneal epithelial cells. J Ocul Pharmacol Ther. 2010;26(3):259-263.

19. Hommer A, Mohammed RO, Burchert M, Kimmich F. IOP-lowering efficacy and tolerability of preservative-free tafluprost $0.0015 \%$ among patients with ocular hypertension or glaucoma. Curr Med Res Opin. 2010;26(8):1905-1913.

20. Cha SH, Lee JS, Oum BS, Kim CD. Corneal epithelial cellular dysfunction from benzalkonium chloride (BAC) in vitro. Clin Exp Ophthalmol. 2004;32(2):180-184.

21. Debbasch C, Brignole F, Pisella PJ, Warnet JM, Rat P, Baudouin C. Quaternary ammoniums and other preservatives' contribution in oxidative stress and apoptosis on Chang conjunctival cells. Invest Ophthalmol Vis Sci. 2001;42(3):642-652.

22. Epstein SP, Ahdoot M, Marcus E, Asbell PA. Comparative toxicity of preservatives on immortalized corneal and conjunctival epithelial cells. J Ocul Pharmacol Ther. 2009;25(2):113-120.

23. Champeau E, Edelhauser H. Effect of ophthalmic preservatives on the ocular surface: conjunctival and corneal uptake and distribution of benzalkonium chloride and chlorhexidine digluconate. In: Holly F, Lamberts D, MacKeen D, editors. The Preocular Tear Film in Health, Disease, and Contact Lens Wear. Lubbock, TX: Dry Eye Institute, Inc; 1986.

24. Margo CE. The placebo effect. Surv Ophthalmol. 1999;44(1): 31-44.

25. Nichols KK, Nichols JJ, Mitchell GL. The lack of association between signs and symptoms in patients with dry eye disease. Cornea. 2004; 23(8):762-770.

26. Tsai JC, McClure CA, Ramos SE, Schlundt DG, Pichert JW. Compliance barriers in glaucoma: A systematic classification. J Glaucoma. 2003;12(5):393-398.

27. Lewis RA, Katz GJ, Weiss MJ, et al. Travoprost $0.004 \%$ with and without benzalkonium chloride: A comparison of safety and efficacy. J Glaucoma. 2007;16(1):98-103.
Clinical Ophthalmology

\section{Publish your work in this journal}

Clinical Ophthalmology is an international, peer-reviewed journal covering all subspecialties within ophthalmology. Key topics include: Optometry; Visual science; Pharmacology and drug therapy in eye diseases; Basic Sciences; Primary and Secondary eye care; Patient Safety and Quality of Care Improvements. This journal is indexed on

\section{Dovepress}

PubMed Central and CAS, and is the official journal of The Society of Clinical Ophthalmology (SCO). The manuscript management system is completely online and includes a very quick and fair peer-review system, which is all easy to use. Visit http://www.dovepress.com/ testimonials.php to read real quotes from published authors. 